\title{
Erratum to: Cation Distribution and Magnetic Properties of Polycrystalline Hexagonal $\mathrm{BaFe}_{12-x} \mathrm{Sn}_{x} \mathrm{O}_{19}$ Ferrites
}

\author{
V. G. Kostishin ${ }^{a, *}$, V. V. Korovushkin ${ }^{a}$, K. V. Pokholok ${ }^{b}$, A. V. Trukhanov ${ }^{a}$, I. M. Isaev ${ }^{a}$, \\ A. Yu. Mironovich ${ }^{a}$, and M. A. Darwish ${ }^{a}$ \\ ${ }^{a}$ National University of Science and Technology "MISIS”, Moscow, 119991 Russia \\ ${ }^{b}$ Moscow State University, Moscow, 119991 Russia \\ *e-mail:drvgkostishyn@mail.ru \\ Received January 12, 2022; revised January 12, 2022; accepted January 12, 2022
}

DOI: $10.1134 / \mathrm{S} 1063783421130011$

The surname of the seventh author should read as follows:

M. A. Darwish

The original article can be found online at

https://doi.org/10.1134/S1063783421100176 